\section{Revista Brasileira de Administração Científica}

Brazilian Journal of Scientific Administration

Abr a Jun 2021 - v.12 - n.2

\title{
A rota do Blockchain: um panorama das pesquisas
}

Este artigo tem por objetivo mapear o perfil das pesquisas sobre Blockchain no período de 2015 a 2019. Este estudo foi conduzido por meio de uma pesquisa bibliométrica em 582 artigos publicados nos principais meios de publicação da pesquisa científica em administração, no período de 2015 a 2019 na base de dados Web of Science. Entre os principais resultados indicam que o interesse pelo assunto é crescente de forma exponencial, saltando de 0 artigos em 2015 para 389 em 2019. Os países de origem que mais apresentaram produção científica no período foi a China, seguidos dos EUA. Com relação à área de pesquisa que mais explora a tecnologia Blockchain apresenta-se as Ciências da Computação, seguida por Engenharia.

Palavras-chave: Blockchain; Bibliometria; Tecnologia.

\section{The Blockchain route: an overview of research}

This paper intend to map the profile of research about Blockchain in the period from 2015 to 2019 . This study was conducted through a bibliometric research in 582 articles published in the mains periodics of publication of scientific research in administration, in the Web of Science database. Among the main results indicate that interest in the subject is growing exponentially, jumping from 0 articles in 2015 to 389 in 2019. The countries of origin that presented the most scientific production in the period was China, followed by the USA. Regarding the research area that most explores Blockchain technology, Computer Science is presented, followed by Engineering.

Keywords: Blockchain; Bibliometry; Technology.

\section{Topic: Inovação Tecnológica}

Reviewed anonymously in the process of blind peer.
Received: $17 / 04 / 2021$

Approved: 10/06/2021

Jessica de Castro (iD

Universidade Estadual do Centro-Oeste, Brasil

http://lattes.cnpq.br/9309941026540112

http://orcid.org/0000-0003-1263-6252

jeessicacastro@hotmail.com

Referencing this:

CASTRO, J.. A rota do Blockchain: um panorama das pesquisas. Revista Brasileira de Administração Científica, v.12, n.2, p.339-346, 2021. DOI: http://doi.org/10.6008/CBPC2179-684X.2021.002.0027 


\section{INTRODUÇÃO}

Blockchain é uma tecnologia de gerenciamento de dados, que foi criada inicialmente como suporte das transações que envolvem a criptomoeda denominada Bitcoin. O formato de funcionamento dessa tecnologia, consiste em um modelo de transações descentralizadas. As primeiras informações a respeito da tecnologia foram expressas em 2008 por Nakamoto (NAKAMOTO, 2008), a partir desta publicação novos interesses de utilização da tecnologia foram surgindo, além das criptomoedas. O motivo pelo qual a tecnologia começou a ganhar espaço no mundo corporativo, pauta-se em suas qualidades evidenciadas como: segurança, anonimato e integridade de dados sem qualquer interferência de terceiros no controle das transações (GREVE et al., 2018; FERREIRA et al., 2017).

A tecnologia Blockchain se trata de um sistema, no qual adota um formato de gestão compartilhada e descentralizada. Esse sistema se dá por meio de uma rede peer-to-peer. Assim, todos os participantes, comummente chamados de 'nós' da rede, possuem a responsabilidade de armazenar as informações e manter a base de dados por meio das validações.

As principais características que definem essa tecnologia são a segurança das operações, descentralização de armazenamento/computação, integridade de dados e imutabilidade de transações. A rede só é capaz de acrescentar informações, não é possível qualquer alteração nos dados já alimentados na rede (WRIGHT et al., 2015). A primeira aparição da Tecnologia Blockchain, se deu em 2008 por meio de uma publicação assinada por Nakamoto. A tecnologia se popularizou e ficou conhecida a partir da utilização pela criptomoeda Bitcoin (NAKAMOTO, 2008).

Posteriormente começaram-se as especulações sobre a utilização dessa tecnologia em outros modelos de negócio que fossem além das transações financeiras, iniciaram-se portanto, a exploração da tecnologia em novos mercados como o, gerenciamento de propriedade intelectual (VELLA et al., 2018), Internet das Coisas (LI et al., 2018; GROMOVS et al., 2017), na gestão pública (ATZORI, 2015) entre outras aplicações possíveis.

A constante evolução tecnológica tem tornado o ambiente organizacional mais complexo e tem exigido que as instituições se adequem e insiram-se neste ambiente de forma rápida e continua. Na medida em que o uso das mais diversas tecnologias disponíveis no mercado hoje cresce, crescem também as pressões externas para que as instituições passem a fazer uso de novas tecnologias.

Nesta perspectiva, esta pesquisa busca analisar como a utilização de uma tecnologia, denominada Blockchain, aderida por um banco brasileiro, pode interferir nas estruturas de governança e nos custos de transação.

No Brasil, o assunto ainda não é de domínio geral, em uma pesquisa realizada pela Federação das Indústria do Estado de São Paulo (2018) 64,4\% das empresas nunca ouviram falar da tecnologia Blockchain. No entanto, algumas empresas, já estão adotando ou analisando a viabilidade de utilização da tecnologia Blockchain em suas atividades, um exemplo, é o caso do Banco Central do Brasil que vem desenvolvendo pesquisas desde 2016 sobre a possibilidade de aplicação em suas transações. 


\section{REVISÃO TEÓRICA}

\section{Blockchain}

Blockchain é uma tecnologia de gerenciamento de dados cujo funcionamento consiste em transações descentralizadas, sendo desenvolvida para a criptomoeda Bitcoin no contexto de pagamento digital. 0 interesse na tecnologia blockchain tem aumentado desde que a ideia foi cunhada em 2008. As razões para o interesse na tecnologia blockchain são seus atributos centrais que fornecem segurança, anonimato e integridade de dados sem qualquer interferência de terceiros no controle das transações. Até agora, o blockchain tem atraído bastante atenção da indústria de serviços financeiros, mas a tecnologia pode ser adaptada para qualquer indústria onde seja necessário registrar, confirmar e transferir qualquer tipo de contrato ou propriedade (FERREIRA et al., 2017).

Em 2008, tornou-se público um artigo técnico contendo os princípios de funcionamento de uma criptomoeda denominada Bitcoin, cuja proposta era a criação de uma moeda digital mundial que funcionasse em uma rede peer-to-peer e que permitisse o envio de pagamentos online de forma totalmente segura, sem o envolvimento de instituições financeiras para todos os participantes da rede. A autoria desse artigo é anônima e está sob o pseudônimo de Satoshi Nakamoto (NAKAMOTO, 2008).

A tecnologia Blockchain pode ser entendida de várias formas. Em linhas gerais, pode-se dizer que se trata de um sistema distribuído de base de dados em log, mantido e gerido de forma compartilhada e descentralizada (através de uma rede peer-to-peer, P2P), na qual todos os participantes são responsáveis por armazenar e manter a base de dados. A tecnologia foi construída tendo em mente quatro principais características arquiteturais: segurança das operações, descentralização de armazenamento/computação, integridade de dados e imutabilidade de transações. O blockchain é mantido simultaneamente por todos os nós da rede P2P, não existindo local principal ou preferencial para armazenamento de uma base de dados original. Todo nó tem a sua réplica da base de dados, e todas são mantidas integras, consistentes e sincronizadas pelos protocolos de consenso (ZALAN, 2018; DAVIDSON et al., 2016; AITZHAN et al., 2016; KYPRIOTAKI et al., 2015).

A tecnologia Blockchain apresenta condições de proporcionar reconfigurações nos formatos de interação entre indivíduos, seja no âmbito político, organizacional, e até mesmo na sociedade em geral. Permite realizar transações sem a necessidade de intermediários de maneira mais rápida, e mais segura. Este formato de interação pode ocasionar mudanças inclusive nos pilares que sustentam os sistemas políticos e os modelos de governança existentes, pondo em causa o papel tradicional do Estado e das instituições centralizadas. Defensores do Blockchain defendem que a substituição das funções tradicionais do estado tornar-se-iam mais eficazes, se substituídas por serviços baseados em Blockchain e plataformas decentralizadas de código aberto. Nessa inserção da tecnologia Blockchain nos processos haveria o incentivo à criação de seus próprios sistemas de governança, nos quais a centralização, a coerção e as hierarquias seriam substituídas por mecanismos de consenso distribuído (ATZORI, 2015).

Uma visão tradicional das estruturas de governança organizacional e política enfatiza a centralização 
e a hierarquia, com vários graus de decisões rígidas de comando e controle de cima para baixo fazendo as regras estabelecidas. O Blockchain indica um modelo de coordenação mais descentralizado, abordando os problemas das estruturas de governança tradicionais pautadas na centralidade, o primeiro deles seria o dilema entre a relação que envolve principal e agente, e o segundo o volume significativo dos custos de transação.

A tecnologia Blockchain permite imaginar um contexto em que os contratos são incorporados ao código digital e armazenados em bancos de dados compartilhados e transparentes, onde são protegidos contra exclusão, adulteração e revisão. Considerando o acesso à informação, a rapidez e a segurança do processo, tem-se que intermediários, tais como, corretores, advogados, banqueiros entre outros, podem não ser mais necessários, organizações de indivíduos, máquinas e logaritmos transacionariam livremente e interagiriam com pouco atrito (IANSITI et al., 2017).

O Bitcoin pode ser considerado como o primeiro caso de uso de Blockchain (IANSITI et al., 2017). Os blockchains não estão apenas alimentando moedas digitais. Eles também são permitindo a criação de contratos inteligentes, um dos primeiros verdadeiramente disruptivos avanços tecnológicos para a prática da lei desde a invenção da impressora de impressão. Usando um banco de dados distribuído, como o Blockchain, as partes podem confirmar que um evento ou condição ocorreu de fato sem a necessidade de um terceiro.

Contrapondo ao formato tradicional das organizações que considera as decisões de maneira centralizada, esse formato descentralizado dispõe que o processo pode ocorrer por meio de codificação sem a necessidade para qualquer parte centralizada confiável gerir o processo (WRIGHT et al., 2015).

A transição para uma maior descentralização pode não só impactar a implementação ou aplicação de leis, mas também como governamos os negócios organizações e sociedade em geral. Aplicações baseadas em Blockchain apresentam uma promessa genuína para novos tipos de inovações escaláveis em governança e desenho institucional, onde os ideais para uma corrupção livre e eficaz a democracia social pode se tornar realidade (WRIGHT et al., 2015).

Embora originalmente concebido como ferramentas de desintermediação, os ecossistemas de Blockchains são caracterizados por uma grande quantidade de terceiros e negócios lucrativos oferecendo serviços de intermediação, com fortes assimetrias de informação e poder entre desenvolvedores e usuários. Na verdade, é comprovado que ele se transforma em formas centralizadas mais sutis ou ocultas (ATZORI, 2015).

Enquanto a mídia tende a descrever Blockchain como uma tecnologia "disruptiva" ou "habilitadora". Atzori (2015), não considera nem um nem outro, mas sim, uma tecnologia transformadora semelhante ao vapor e a eletricidade, que criará novos fundamentos econômicos e sociais.

\section{METODOLOGIA}

A técnica de pesquisa utilizada para este estudo será a bibliométrica, a qual permitirá um levantamento dos estudos que abrangem o tema blockchain e verificar em um período específico como se 
comportaram as publicações acerca do assunto. Para tanto foram realizadas de três etapas. A primeira etapa foi representada pela delimitação das bases de dados a serem consultadas. Pretende-se, portanto, buscar as fontes mais influentes relacionadas ao tema, a fim de possibilitar um retrato relevante da situação a ser pesquisada. Dessa forma, será considerada para a seleção dos trabalhos, os estudos publicados na base de dados Web of Science.

A segunda etapa se caracterizará pela seleção dos artigos conforme a base de dados definida no item anterior. Nesta etapa serão definidos os critérios de busca para seleção dos estudos apropriados à presente intenção de pesquisa. Para tanto serão utilizadas a seguinte palavras-chave: 'Blockchain'.

Na terceira etapa serão definidas e identificadas nos artigos selecionados as variáveis que permitirem delinear um panorama dos estudos sobre blockchain no contexto nacional partir dos seguintes aspectos: a. $\mathrm{O}$ ano de publicação da obra; b. País de origem da pesquisa; c. Instituição de Ensino Superior vinculadas às pesquisas; d. A autoria e coautoria dos trabalhos analisados; e. Área de estudo na qual o tema se insere.

\section{RESULTADOS E DISCUSSÃO}

A coleta de dados se deu por meio da busca dos artigos disponíveis na base de dados Web of Science. A busca se deu por meio da palavra-chave 'Blockchain' constante nos títulos dos artigos disponíveis. Outro filtro utilizado foi a data que se concentrou entre 2015 e 2019. A busca retornou um total de 3.973 documentos. Refazendo a busca e utilizando o filtro 'artigos' para selecionar o tipo de documento, a busca resultou em 1298 artigos. Ainda, desses 1298 artigos, 582 eram de acesso aberto, os quais possibilitaram a realização da pesquisa. A tabela 1 mostra o desenvolvimento nitidamente crescente nos estudos relacionados ao Blockchain.

Tabela 1: Ano de Publicação.

\begin{tabular}{lll} 
& Observações & \\
\hline Ano & Frequência & $\%$ \\
\hline 2019 & 389 & $66.84 \%$ \\
2018 & 148 & $25.43 \%$ \\
2017 & 39 & $6.70 \%$ \\
2016 & 6 & $1.03 \%$ \\
2015 & 0 & $0.00 \%$ \\
\hline Total & 582 & 1
\end{tabular}

Pode-se perceber, que de fato, o tema Blockchain vem se consolidando e chamando fortemente a atenção dos pesquisadores. A Tabela 1, mostra um salto de 0 pesquisas em 2015 para 30 em 2017, e mais expressivamente um salto para 389 durante o ano de 2019. Embora a tecnologia tenha surgido em 2008 (NAKAMOTO, 2008), nota-se um intervalo de tempo significativo entre o surgimento da tecnologia e o interesse de estudos nesta área. A Tabela 2 evidencia o país de origem de realização da pesquisa. O país que se destaca quanto a produção de artigos científicos sobre o Blockchain é China.

De acordo com a Tabela 2, China vem liderando o ranking das pesquisas acadêmicas no campo da tecnologia Blockchain com a produção de 207 artigos neste intervalo de 5 anos, lembrando que a maior produção ocorreu no último ano. Em segundo lugar no ranking de produção acerca do tema estudado vem os Estados Unidos com o montante de 98 artigos e em seguida a Coreia do Sul. Cabe ressaltar que foram 
destacados os 5 países mais produtivos dada a sua relevância no volume de produção, no entanto, além dos 5 apresentados na Tabela 2, foram identificados nos artigos selecionados 69 outros países dos quais houve produção acadêmica relacionada ao tema Blockchain. Na sequência a Tabela 3 apresenta as principais organizações que estão vinculadas as pesquisas relacionadas a tecnologia Blockchain:

Tabela 2: País de origem da pesquisa.

\begin{tabular}{ll}
\hline Países & Quantidade \\
\hline China & 207 \\
EUA & 98 \\
Coreia do Sul & 65 \\
Inglaterra & 56 \\
Austrália & 27 \\
\hline
\end{tabular}

Tabela 3: Instituições de Ensino Superior vinculadas às pesquisas.

\begin{tabular}{ll}
\hline IES/Organizações & Quantidade \\
\hline Beijing University of Posts Telecommunications & 17 \\
Chinese Academy of Sciences & 15 \\
University of Electronic Science Technology of China & 15 \\
University of Texas System & 11 \\
Xidian University & 10 \\
\hline
\end{tabular}

Identifica-se na Tabela 3 que Beijing University of Posts Telecommunications lidera o volume de pesquisas realizadas afiliadas a uma instituição, localizada em Pequim, assim como as segundas e terceiras colocadas da tabela Chinese Academy of Sciences e University of Electronic Science Technology of China com a produção de 15 artigos cada, estão localizadas na China, corroborando com os dados apresentados na Tabela 2 sobre a expressiva produção do país Chines. Além delas, a Xidian University também está localizada na China, reforçando e justificando ainda mais a produção deste país. Em quarto lugar no ranking aparece University of Texas System com um total de 11 artigos publicados.

Assim como não foi possível apresentar todos os países devido ao volume expressivo de diferentes origens, no caso das Instituições as quais os autores são afiliados ocorreu a mesma situação. Ao todo foram identificadas 856 instituições de ensino superior diferentes e, portanto, não foi possível descrevê-las em sua totalidade nesta sessão.

A seguir tem-se os principais nomes por trás dessa crescente produção, a Tabela 4, relaciona os principais autores que realizam pesquisas relacionadas ao tema Blockchain.

Tabela 4: Principais autores.

\begin{tabular}{ll}
\hline Autores & Quantidade \\
\hline PARK, J. H. & 207 \\
Wang, J. & 98 \\
Guizani, M. & 65 \\
Salah, K. & 56 \\
Zhang, Y & 27 \\
\hline
\end{tabular}

Evidenciam-se nesta sessão Park, J. H. com uma relevante produção de 207 artigos no período analisado, Wang, J com 98 artigos no período seguido por Guizani, M. assinando 65 dos artigos produzidos. A maioria dos artigos apresentava coautoria de 2, até 5 coautores, dessa forma, assim como nas demais tabelas, não foi possível relacionar todos os autores identificados na pesquisa. Neste caso, ainda mais expressivo, a pesquisa contou com um total de 1739 autores, além dos apresentados na Tabela 4. Abordou- 
se, portanto, os nomes mais relevantes de acordo com maior volume de produção no período.

Por fim, na Tabela 5, são apresentadas as principais áreas de interesse, nas quais as pesquisas são classificadas.

Tabela 5: Área de classificação da pesquisa.

\begin{tabular}{ll}
\hline Área de pesquisa & Quantidade \\
\hline Ciências da Computação & 17 \\
Engenharia & 15 \\
Telecomunicações & 15 \\
Negócios e Economia & 11 \\
Química & 10 \\
\hline
\end{tabular}

Embora tecnologia Blockchain, tenha se popularizado a partir da utilização pela criptomoeda Bitcoin (NAKAMOTO, 2008), está temática é altamente multidisciplinar. A utilização dessa tecnologia vem ganhando espaços em outros modelos de negócio que atuam além das transações financeiras, tais como, a exploração da tecnologia em novos mercados como o, gerenciamento de propriedade intelectual (VELLA et al., 2018), Internet das Coisas (LI et al., 2018; GROMOVS et al., 2017), na gestão pública (ATZORI, 2015) entre outras aplicações possíveis.

Desse modo, percebemos destaque nas pesquisas relacionadas a área de Ciências da Computação, e isso faz sentido, considerando a necessidade técnica com a qual o Blockchain é construído e utilizado. E é somente a partir da parte técnica computacional que pode ser utilizado em qualquer outro segmento. Além das Ciências da Computação, áreas como Engenharia, Telecomunicações e Negócios vem sendo exploradas a partir das pesquisas sobre Blockchain. Em adição, considerando ainda a limitação de espaço para listar todas as áreas, foi identificado que as áreas de Medicina, Sociologia e Ciências Ambientais são as menos exploradas quando se trata dessa tecnologia, tais áreas, dispuseram de apenas 1 artigo cada, no período analisado. Ao todo foram identificadas 48 classificações de áreas distintas nas pesquisas observadas.

\section{CONCLUSÕES}

Ao longo do estudo se evidenciou a existência de um crescente em termos quantitativos nas publicações que versam sobre Blockchain durante o período analisado, demonstrando o interesse pelo tema por parte dos pesquisadores. Esse contexto, vem ganhando mais expressividade a partir da sua popularização, bem como a descoberta de aplicabilidade nas mais diversas áreas.

Este estudo se propôs analisar bibliométricamente publicações durante o período de 2015 a 2019 nos principais periódicos nacionais e internacionais listados na base de dados Web of Science, a fim de demonstrar como essa tecnologia tão disruptiva e inovadora vem sendo estudada no contexto mundial. A amostra encontrou inicialmente 3973 documentos e após os balizamentos e descartes de documentos que não se enquadravam como artigos, e de artigos que não são abertos a consulta, restaram para a base de dados analisada 580 artigos. A publicação dos artigos se concentra significativamente nos anos mais recentes, o que evidencia um crescente interesse no tema pesquisado.

Tanto os principais autores quanto as principais instituições de ensino de acordo com o volume de produção, destacadas na pesquisa são de origem Chinesa, construindo com a informação de que a China é o 
país com maior volume de produção de artigos acadêmicos a partir dos dados analisados.

Este trabalho apresenta a limitação de trabalhar com apenas uma base de dados e também o fato de considerar apenas os artigos que estavam classificados como 'abertos' na plataforma, não compondo a amostra, mais da metade dos artigos inicialmente encontrados sobre o tema. A relevância reside em destacar o crescente interesse por um tema que se demonstra emergente e extremamente atual no qual, muitas lacunas podem ainda ser exploradas.

\section{REFERÊNCIAS}

AITZHAN, N. Z.; SVETINOVIC, D.. Security and Privacy in Decentralized Energy Trading through Multi-Signatures, Blockchain and Anonymous Messaging Streams. IEEE Transactions on Dependable and Secure Computing, v.15, n.5, 2016. DOI: http://doi.org/10.1109/TDSC.2016.2616861

ATZORI, M.. Blockchain technology and decentralized governance: is the state still necessary?. 2015. DOI: http://dx.doi.org/10.2139/ssrn.2709713

DAVIDSON, S.; FILIPPI, P.; POTTS, J.. Economics of Blockchain. 2016. DOI:

http://dx.doi.org/10.2139/ssrn.2744751

FERREIRA, J. E.; PINTO, F. G. C.; SANTOS, S. C.. Estudo de mapeamento sistemático sobre as tendências e desafios do Blockchain. Revista Gestão, v.15, p.108-117, 2017.

GREVE, F.; SAMPAIO, L.; ABIJAUDE, J.; COUTINHO, A.; VALCY, I.; QUEIROZ, S.. Blockchain e a Revolução do Consenso sob Demanda. In. SIMPÓSIO BRASILEIRO DE REDES DE COMPUTADORES E SISTEMAS DISTRIBUÍDOS (SBRC), 36. Anais. Campos do Jordão, 2018.

GROMOVS, G.; LAMMI, M.. Blockchain and internet of things require innovative approach to logistics education. Transport Problems: an International Scientific Journal, v.12, p.23-35, 2017.
IANSITI, M.; LAKHANI, K. R.. The Truth about Blockchain: It will take years to transform business, but the journey begins now. Harward Business Review, p.118-127, 2017.

KYPRIOTAKI, K. N.; ZAMANI, E. D.; GIAGLIS, G. M.. From bitcoin to decentralized autonomous corporations: extending the application scope of decentralized peer-topeer networks and blockchains. In: INTERNATIONAL CONFERENCE ON ENTERPRISE INFORMATION SYSTEMS ICEIS, 17. Anais. Barcelona, 2015. p.284-290. DOI: http://doi.org/10.5220/0005378402840290

LI, Z.; KANG, J.; YU, R.; YE, D.; DENG, Q.; ZHANG, Y.. Blockchain do consórcio para o comércio seguro da energia na Internet industrial das coisas. IEEE Transactions on Industrial Informatics, v.14, n.8, p.3690-370, 2018.

NAKAMOTO, S.. Bitcoin: a peer-to-peer electronic cash system. White Paper, 2008.

VELLA, D.; FALZON, M.; CASSAR, T.; VALENZIA, A.. A aplicabilidade da Blockchain ao gerenciamento de propriedade intelectual. The Licensing Journal, v.38, n.1, 2018.

WRIGHT, A.; FILIPPI, P.. Decentralized Blockchain Thechnology and the Rise of Lex Cryptographia. 2015.

ZALAN, T.. Born global on blockchain. Review of International Business and Strategy, v.28, n.1, p.19-34, 2018. DOI: https://doi.org/10.1108/RIBS-08-2017-0069

A CBPC - Companhia Brasileira de Produção Científica (CNPJ: 11.221.422/0001-03) detém os direitos materiais desta publicação. Os direitos referem-se à publicação do trabalho em qualquer parte do mundo, incluindo os direitos às renovações, expansões e disseminações da contribuição, bem como outros direitos subsidiários. Todos os trabalhos publicados eletronicamente poderão posteriormente ser publicados em coletâneas impressas sob coordenação da Sustenere Publishing, da Companhia Brasileira de Produção Científica e seus parceiros autorizados. Os (as) autores (as) preservam os direitos autorais, mas não têm permissão para a publicação da contribuição em outro meio, impresso ou digital, em português ou em tradução. 